\title{
Bayesian approach for uncertainty quantification in water quality modelling: The influence of prior distribution
}

\author{
Gabriele Freni ${ }^{\mathrm{a}, *}$, Giorgio Mannina ${ }^{\mathrm{b}}$ \\ a Facoltà di Ingegneria ed Architettura, Università di Enna "Kore", Cittadella Universitaria, 94100 Enna, Italy \\ ${ }^{\mathrm{b}}$ Dipartimento di Ingegneria Idraulica ed Applicazioni Ambientali, Università di Palermo, Viale delle Scienze, 90128 Palermo, Italy
}

\section{A R T I C L E I N F O}

\section{Article history:}

Received 14 April 2010

Received in revised form 17 July 2010

Accepted 28 July 2010

This manuscript was handled by

Geoff Syme, Editor-in-Chief

\section{Keywords:}

Bayesian approach

Prior knowledge

Uncertainty assessment

Urban stormwater quality modelling

\begin{abstract}
S U M M A R Y
Mathematical models are of common use in urban drainage, and they are increasingly being applied to support decisions about design and alternative management strategies. In this context, uncertainty analysis is of undoubted necessity in urban drainage modelling. However, despite the crucial role played by uncertainty quantification, several methodological aspects need to be clarified and deserve further investigation, especially in water quality modelling. One of them is related to the "a priori" hypotheses involved in the uncertainty analysis. Such hypotheses are usually condensed in "a priori" distributions assessing the most likely values for model parameters. This paper explores Bayesian uncertainty estimation methods investigating the influence of the choice of these prior distributions. The research aims at gaining insights in the selection of the prior distribution and the effect the user-defined choice has on the reliability of the uncertainty analysis results. To accomplish this, an urban stormwater quality model developed in previous studies has been employed. The model has been applied to the Fossolo catchment (Italy), for which both quantity and quality data were available. The results show that a uniform distribution should be applied whenever no information is available for specific parameters describing the case study. The use of weak information (mostly coming from literature or other model applications) should be avoided because it can lead to wrong estimations of uncertainty in modelling results. Model parameter related hypotheses would be better dropped in these cases.
\end{abstract}

(c) 2010 Elsevier B.V. All rights reserved.

\section{Introduction}

Complex environmental models generally demand adequate input for their implementation that has to be obtained by large gathering campaigns. These latter are extensive and generally require large economic as well as human resources, which are not always available. Databases are often of limited size and calibration problems connected with the lack of data, especially regarding water quality, often have to be faced by the modeller. Owing to these problems, model uncertainty may be relevant, and its quantification is imperative for assessing model reliability. However the state of knowledge on uncertainties in urban drainage models is still piecemeal, some studies are reported in literature, which are mainly aimed at parameter screening for supporting model calibration (Harremoës and Madsen, 1999; Kanso et al., 2004; Freni et al., 2008; Thorndahl et al., 2008; Willems, 2008; Willems, 2000; Deletic et al., 2009).

A modeller's usual objective is to reduce to zero the gap between the simulated and the observed system behaviour, but this

\footnotetext{
* Corresponding author. Tel.: +39 0916657700; fax: +390916657749.

E-mail addresses: gabriele.freni@unikore.it (G. Freni), mannina@idra.unipa.it (G. Mannina).
}

goal is generally impossible owing to the unavoidable uncertainties inherent in any modelling procedure. Uncertainty analysis in environmental modelling can provide information on the 'goodness' of a result and enables us to pin down the sources of error in the modelling process, their relative importance when propagated to the model outputs, and consequently the priorities for model improvement (Willems, 2008). Furthermore, without uncertainty quantification, complementary to a measure of the goodness of a result, it is difficult to judge the reliability of the results regarding the value of the impact for water engineering decision support (Reichert, 1997; Refsgaard et al., 2005; Schaarup-Jensen et al., 2005; Krysanova et al., 2007).

The uncertainty of a model result can be stated by giving a range (or a band) of values that are likely to enclose the true value of a specific simulated variable: lower uncertainty is connected with stricter uncertainty bands; larger bands are caused by highly uncertain models. Using the concept of uncertainty, the best model is the one able to correctly simulate a specific variable while minimising the width of the uncertainty bands. Previous research on uncertainty analysis in environmental models has generally addressed three types of uncertainties (Deletic et al., 2009; Freni et al., 2008; Willems, 2008; Lindenschmidt et al., 2007): 
- Structural uncertainty: uncertainty due to the structure of the model, which includes the equations and algorithms used for the simulations and coupling of models.

- Input data uncertainty: uncertainty in the data used as boundary and initial conditions in the model.

- Parameter uncertainty: uncertainties in model parameters and uncertain information used for parameter estimation.

Structural uncertainty is due to the assumptions and simplifications in the model and the application of the model under conditions that are not quite consistent with the model design. Structural uncertainty is very difficult to quantify, and only a few attempts at doing so are found in the literature (Lindenschmidt et al., 2007).

Uncertainty in model input occurs because of changes in natural conditions, limitations of measurements, and lack of data (Beck, 1987). One way to simulate the effect of input uncertainties is to use random distributions to describe the error in the input data, rather than the conventional form of fixed values.

In urban drainage models, several authors have estimated the uncertainties involved (Freni et al., 2008, 2009a; Thorndahl et al., 2008; Willems, 2008; Kanso et al., 2005; Willems and Berlamont, 2002) but few studies have focused on water quality modelling, which, in comparison with the modelling of quantity phenomena, involves several types of additional uncertainties in pollution load inputs and sewer quality processes (Thorndahl and Willems, 2008; Willems, 2008). These additional uncertainties are important given the high complexity of sewer water quality processes or environmental processes in general, as well as the consequent lack of knowledge about and the limited amount of sewer quality sampling data (Beck, 1987; Ashley et al., 2004; Mannina, 2006; Willems, 2009). In this context, the uncertainty of a simplified urban drainage model developed in previous studies has been evaluated. Different approaches can be used for uncertainty analysis. In this study, a Bayesian approach coupled with Monte Carlo analysis has been used (Bayes, 1763). The Bayesian approach expresses uncertainties in the model parameters in terms of probability. Parameter uncertainty is quantified first by introducing a prior probability distribution, which represents historical or expert information before any new data are collected. The main objective of this paper is to quantify the effect of the choice of the prior distribution on the uncertainty assessment. Analysing both water quantity and quality aspects, the modelling application will be carried out in data scarcity conditions, thus increasing the probable influence of prior knowledge. The model has been applied to the Fossolo case study (Italy), for which quantity and quality data are available.

\section{Materials and methods}

\subsection{The urban drainage model}

Details about the urban water quality drainage model employed in the present study can be obtained from previous studies (Mannina, 2005; Mannina and Viviani, 2010) a brief description is discussed in the following. The model is a simplified conceptual model that is composed of two connected modules: a hydrological and hydraulic module and a mass transfer module. The former enables to calculate the hydrographs at the sewer outlet, whereas the mass transfer module quantifies the pollutographs. The hydrological - hydraulic module evaluates the net rainfall from the measured hyetograph considering a loss function (taking into account surface storage and soil infiltration). Once evaluated the net rainfall, the hydrograph in sewer is assessed by means of a cascade of a linear reservoir and a channel (representing the catchment) and a linear reservoir (representing the sewer network). With regards to the water quality processes, the model simulates the build-up of the pollutant loads considering the accumulation of the pollutants in the catchment surfaces and in sewer during the dry weather period. The wash-off and erosion of sewer sediments are simulated during the wet weather for the assessment of sewer pollutograph. Further, the propagation of solids in the sewer network is simulated considering also their sedimentation and re-suspension. Concerning the sewer sediments, the model considers the sediment transformation in sewers, taking into account their cohesive-like behaviour related to organic substances and to the physical-chemical changes during the sewer transport (Ristenpart, 1995). In particular, the transport equation proposed by Parchure and Mehta (1985) jointly with the sediment bed structures proposed by Skipworth et al. (1999) was considered to simulate the sediment erosion rate. The pollutographs at the inlet and outlet of the sewer system have been evaluated by hypothesizing the complex catchment sewer network as a reservoir and by introducing a transport capacity of the flow. This latter takes into account the different types of sewer sediment transport (i.e. bed load and suspended load).

\subsection{Model uncertainty assessment using the Bayesian Monte Carlo approach}

Some uncertainty analysis techniques use the notion of probability to represent uncertainty or partial knowledge of a system that may be deterministic. In such approaches, model parameters are associated with a probability distribution stating the specific range within which the "real" (or calibrated) parameter value may be contained (Reichert and Omlin, 1997).

The Bayesian method uses parameter probability distributions for representing the operator knowledge before and after the application of the model to a specific data set. The prior probability distribution $P(\theta)$ of the model parameter $\theta$ is defined as the historical or expert information before collecting any new data. The Bayes' theorem on conditional probabilities (Bayes, 1763) is used to update a prior probability distribution of model parameters to the posterior distribution that should include the additional information provided by measurements $(D)$ and by the model application to the analysed system. The Bayesian update can be carried out by means of the definition of conditional probability as:

$P(\theta \mid D)=\frac{P(D \mid \theta) P(\theta)}{\int P(D \mid \theta) P(\theta) d \theta}$

where the posterior parameter distribution is computed as a function of the prior distribution, $P(\theta)$ and the conditional probability for the measured data given the parameter vector $\theta, P(D \mid \theta)$ The distribution function $P(D \mid \theta)$ is often referred to as the likelihood function of the model. Eq. (1) cannot be generally evaluated by analytical approach. This is mainly due to the complexity of the integral at the denominator. For this reason a numerical estimation has been carried out in the present study considering that a discrete number of parameter sets was investigated in the Monte Carlo Analysis. The selection of the appropriate number of Monte Carlo simulations to obtain a good representation of the posterior distributions is a relevant task. In the present study, this sample dimension was selected, verifying that posterior distributions were not affected by any bias linked to the number of Monte Carlo simulations. The analysis started with 500 simulations and this number was increased, by steps of 100 simulations; the analysis is stopped when the posterior distributions obtained in two following steps are characterised by differences lower than 0.01 in terms of cumulated probability (Bertrand-Krajewski et al., 2002). 
The use of the Bayesian approach to uncertainty analysis has been stimulated by the rigorous theoretical framework and by the possibility of evaluating the impact of new knowledge on model parameter estimates (among others: Bates and Campbell, 2001; Kavetski et al., 2006a,b; Yang et al., 2008). Nevertheless, the Bayesian approach relies on some hypotheses that have to be carefully analysed.

In common applications, the hypothesis is made regarding the normal distribution of the residuals between the model and observations assuming the null average and variance $\sigma_{e}^{2}$. According to such hypothesis, $P(D \mid \theta)$ can be written in the multiplicative form as:

$P(D \mid \theta)=\prod_{i=1}^{m} \frac{1}{\sqrt{2 \pi \sigma_{e}^{2}}} \exp \left(\frac{\left(D_{i}-Y_{i}\right)^{2}}{-2 \sigma_{e}^{2}}\right)$

where $Y_{i}$ are the modelling responses that correspond to the $m$ available measurements $D_{i}$ of a specific variable (i.e., discharges, concentrations, loads, etc.) at a specific system cross-section. The application of Eq. (2) is based on the hypotheses that residuals are homoscedastic, independent and identically distributed in time. Such hypotheses should be verified especially considering that modelled and measured time series are likely to be auto-correlated. The Gaussianity of residuals may be obtained by means of transformations, such as the Box-Cox one (Box and Cox, 1982). The application of transformations may affect the results of the analysis (Thyer et al., 2002; Gallagher and Doherty, 2007; Chin, 2009) and the Gaussianity of residuals has to be verified after the transformation is applied. In the present study, the Box-Cox transformation was used for allowing the applicability of Eq. (2) in which transformed variables are introduced and $\sigma_{e}^{2}$ is the variance of residuals computed after the transformation (Box and Cox, 1982). The Gaussianity of residuals after the transformation was verified by means of $\chi^{2}$ test with a confidence level equal to 0.05 (Kottegoda and Rosso, 2008).

Another important element that may prevent Bayesian approaches from being objective is the selection of prior parameter distributions that are usually not obtained from physical observations but based on expert judgement.

Because the analysis of this subjective choice of the prior distribution is the aim of the present paper, different distributions have been considered, each assuming various levels of prior knowledge, that will be further discussed in the following paragraph.

The posterior distribution may be used to refine the estimation of model parameters if the method is aimed at calibration, or it can be used directly to calculate probability distributions for model predictions, thus contributing to the estimation of model uncertainty.

This latter application may be summarised in the following steps:

1. An initial local sensitivity analysis is used for limiting the study to only the influential model parameters. Parameters having scarce impact on the model outputs can be neglected, and their values can be assumed constant.

2. Prior parameter distributions are obtained from all available knowledge. Empirical distributions may be obtained from direct measurements (if the parameter is measurable) or by calibration on available data. Theoretical distributions may be assumed by literature or prior knowledge (Jaynes, 1968).

3. Posterior distributions may be populated by applying Eqs. (2) and (1) on model outputs provided by Monte Carlo analysis that can be obtained by totally random sampling or by stratified sampling like Latin Hypercube approach (Iman et al., 1981).

4. The uncertainty bands are calculated using the $5 \%$ and $95 \%$ percentiles of the predicted output posterior distribution. The uncertainty bands should contain the observations, unless of a small amount of data points depending on the adopted percentiles; otherwise, the model structure should be rejected. Wider bands mean higher uncertainty in the estimation of the modelling output and thus lower confidence in the model results; vice versa, smaller bands containing the observations are symptoms of a reliable and robust modelling approach.

Steps $2-4$ can be iterated each time new data are available.

\section{Case study description}

The Fossolo catchment is located in Italy in a residential area nearby Bologna. The catchment is characterised by an independent combined sewer network where there are not contributions coming from the surrounding catchment. Fossolo is characterised by a total area of 40.71 ha, $74.80 \%$ of which is impermeable (approximately 30.45 ha). The catchment is mainly characterised by residential medium density areas with small parks and pervious lots. Some commercial activities are present in the catchment even if their contribution to dry weather flows and polluting loads is not significant. The catchment has approximately 10,000 equivalent inhabitants. The catchment contains roads with a high vehicle flux (about 40,000 vehicles/day) as well as other streets with a low vehicle flux (about 1000 vehicles/day).

The most downstream sewer pipe is characterised by polycentric cross-section, $144 \mathrm{~cm}$ high and $180 \mathrm{~cm}$ wide. Discharge is estimated from water depth measured by an ultrasonic probe placed in the most downstream sewer pipe. A refrigerated automatic sampler with 24 bottles was used for collecting dry weather and wet weather samples. Biological Oxygen Demand (BOD), Chemical Oxygen Demand (COD) and Total Suspended Solids (TSS) were assessed for each sample according to the Standard Methods (APHA, 1995). In the catchment, 12 events have been measured for both quantity and quality aspects (Table 1). The field campaign was conducted by Bologna University (Artina et al., 1997).

\section{Methodology application and analysis of the results}

According to the steps discussed in previous paragraph, an initial local sensitivity analysis was performed to identify the most influential model parameters. To guarantee a consistent analysis that avoids any subjectivity, parameter variation ranges were assumed as the intervals that strictly include the calibrated values obtained by means of all available monitored events (Beven and Binley, 1992). Average sensitivity indices were calculated by perturbing one model parameter at the time and considering the hydrograph peak and volume for quantity model parameters and the pollutograph for quality parameters, in terms of TSS at the sewer system outlet. The sensitivity index of each modelling output can be computed by the following equation:

$S_{i, j}=\frac{\Delta \theta_{j}}{y s_{i}} \frac{\partial y_{i}}{\partial \theta_{j}}$

where $\Delta \theta_{j}$ is the variability range of parameter $\theta_{j}$ depending on prior knowledge and $y s_{i}$ is a reference (or scaling) value for the modelling output variable $y_{i}$ used for preserving the nondimensional nature of the sensitivity function. In the present study, $y s_{i}$ is defined as the average measured value of the $i$ th model output variable. The derivative in Eq. (3) was numerically computed by finite difference method: the range $\theta_{j}$ was divided in 100 equal intervals; the output variables were computed for the extremes of each interval allowing for the calculation of 100 local values of the derivative. As the sensitivity analysis was used for neglecting non-influential parameters, the maximum absolute value of the derivative 
Table 1

Characteristics of 12 rain events used in the application.

\begin{tabular}{lcccc}
\hline & $\begin{array}{l}\text { Total rainfall } \\
(\mathrm{mm})\end{array}$ & $\begin{array}{l}\text { Max intensity } \\
(\mathrm{mm} / \mathrm{h})\end{array}$ & $\begin{array}{l}\text { Duration } \\
(\mathrm{min})\end{array}$ & $\begin{array}{l}\text { ADWP } \\
(\mathrm{h})\end{array}$ \\
\hline Minimum & 2.8 & 12 & 45 & 50 \\
Maximum & 72.2 & 147 & 921 & 402 \\
Mean & 18.9 & 45 & 258 & 167 \\
\hline
\end{tabular}

was used for computing the sensitivity $s_{i, j}$ of the variable $y_{i}$ in the interval $\Delta \theta_{j}$.

When dealing with multiple modelling outputs, the analysis of functions $s_{i, j}$ may be scarcely informative and a more aggregated index may be useful. For this reason, the weighted average sensitivity has been used for initial evaluation of parameters:

$\bar{s}_{j}=\frac{1}{n} \sum_{i=1}^{n} \frac{s_{i, j}}{\max \left(s_{i, j}\right)}$

where $\max \left(s_{i, j}\right)$ is maximum of the $n$ sensitivities derived for each modelling output for the $j$ th model parameter.

The results of the sensitivity analysis are presented in Table 2: six water quality parameters exhibit sensitivity indices distinctively lower than the others. Therefore, these six model parameters were excluded from the following analysis, and their values were kept constant and equal to the calibration values.

From the initial twenty-seven model parameters, twenty-one were considered in the uncertainty analysis. Once the number of model parameters was reduced to the most influential ones, the influence of the prior knowledge on the parameter distributions was calculated. More specifically, three initial knowledge scenarios were considered:

(a) For the first scenario, prior knowledge was based on prior calibration of parameters, which provided empirical distributions (Fig. 1 ).

(b) For the second scenario, no prior knowledge was available, so uniform distributions were applied (Beven and Binley, 1992).

(c) For the last scenario, prior knowledge was based on general considerations. In this case, log-normal distributions were considered for those parameters varying in a wide range (for two or more orders of magnitude), whereas normal distributions were applied for the others (Kuczera and Parent, 1998). The parameters with a normal distribution were computed assuming a median equal to the average value of the parameter variation range and a standard deviation equal to $1 / 6$ of the variation range (so that the probability that a parameter values falls outside the variation range is 0.003 and it can be considered negligible). On the other hand, for the parameters with log-normal distributions, the median of the distribution of the logarithms was assumed to be equal to the average of the parameter logarithms in the variation ranges; the standard deviation of logarithms was taken to be equal to $1 / 6$ of the variation range (expressed in logarithms). According to this hypothesis, parameters have more than $99.7 \%$ of prior probability to fall inside the initial variation range.

In all the scenarios, 1000 Monte Carlo simulations were run for computing posterior distribution in each Bayesian update step. This number was decided applying the methodology described in the previous paragraph and its computational cost is not relevant as the analysis of each scenario was carried out in less than 40 min by means of an ordinary desktop computer. The Bayesian approach was applied after that the simulated and modelled output variables were transformed according to Box-Cox approach. The Gaussianity of residuals was positively verified for all the analysed output variables by means of $\chi^{2}$ test with a level of confidence set to 0.05 .

To investigate the "learning process" (e.g. Mantovan and Todini, 2006), the dataset (consisting of twelve monitored events) was split in two subsets (including six events each), and two subsequent Bayesian updates were carried out. At each step, uncertainty bands were computed for both discharge and TSS concentrations at the sewer system outlet. In the following section, the analysis will be based on graphs obtained for the rainfall event of 25 April 1994 used as reference for comparing results of the different approaches. In particular, the selected event is characterised by an antecedent dry weather period (ADWP) of 9 days and storm duration of approximately $120 \mathrm{~min}$.

The Bayesian learning process is shown clearly in Figs. 2 and 3 for four selected model parameters. In particular, Fig. 2 shows probability distributions for the $\lambda$ and $W_{0}$ parameters that respectively control the channel delay and the initial hydrologic depletions in the water quantity sub-model, while Fig. 3 shows the probability distributions for the parameters ARRA and KSUSP, representing the catchment wash-off coefficient and the solid suspension delay in the sewer system. The following considerations may be drawn from these probability density functions:

Table 2

Sensitivity indices and variation range of model parameters (insensitive parameters in italics).

\begin{tabular}{|c|c|c|c|c|c|}
\hline & Parameter & Symbol & Unit & Variation range & Sensitivity index \\
\hline \multirow[t]{5}{*}{ Water quantity } & Channel constant & $\lambda$ & $\min$ & $0.04-6$ & 0.166 \\
\hline & Initial hydrological losses & $W_{0}$ & $\mathrm{~mm}$ & $0.2-1.5$ & 0.209 \\
\hline & Catchment runoff coefficient & $\Phi$ & - & $0.25-0.57$ & 0.539 \\
\hline & Catchment reservoir constant & $K 1$ & $\min$ & $2-7$ & 0.232 \\
\hline & Sewer reservoir constant & $K 2$ & $\min$ & $2-7$ & 0.212 \\
\hline \multirow[t]{12}{*}{ Water quality } & Daily accumulation rate & ACCU & {$[\mathrm{kg} /(\mathrm{ha} * \mathrm{~d})]$} & $0.4-25$ & 0.214 \\
\hline & decay rate in Alley-Smith model & DISP & {$\left[\mathrm{d}^{-1}\right]$} & $0.4-0.5$ & 0.733 \\
\hline & Wash-off coefficient & ARRA & $\mathrm{mm}^{-\mathrm{Wh}} \mathrm{h}^{(\mathrm{Wh}-1)}$ & $1.1-1.7$ & 0.159 \\
\hline & Wash-off factor & $\mathrm{WH}$ & - & $2.1-2.7$ & 0.307 \\
\hline & Sewer sediment deposition coefficient & Kdep & $h^{-1}$ & $0.01-1.9$ & 0.040 \\
\hline & Maximum sewer sediment height & hedsmax & $m$ & $0.01-0.1$ & 0.062 \\
\hline & Depth of the weak surface layer & $d^{\prime}$ & $\mathrm{cm}$ & $0.06-3$ & 0.097 \\
\hline & Power term describing variation of erosion resistance in the weak layer & b_Skip & - & $0.008-0.75$ & 0.057 \\
\hline & Yield strength at uniform layer & $\overline{T A U C U}$ & $\mathrm{~N} / \mathrm{m}^{2}$ & $4-10$ & 0.096 \\
\hline & Erosion coefficient & $\mathrm{M}$ & $\mathrm{kg} / \mathrm{s}$ & $10-180$ & 0.257 \\
\hline & Sewer suspension delay & KSUSP & $\mathrm{h}$ & $0.04-0.15$ & 0.495 \\
\hline & Sewer bed transport delay & KFONDO & $h$ & $0.006-0.7$ & 0.065 \\
\hline
\end{tabular}



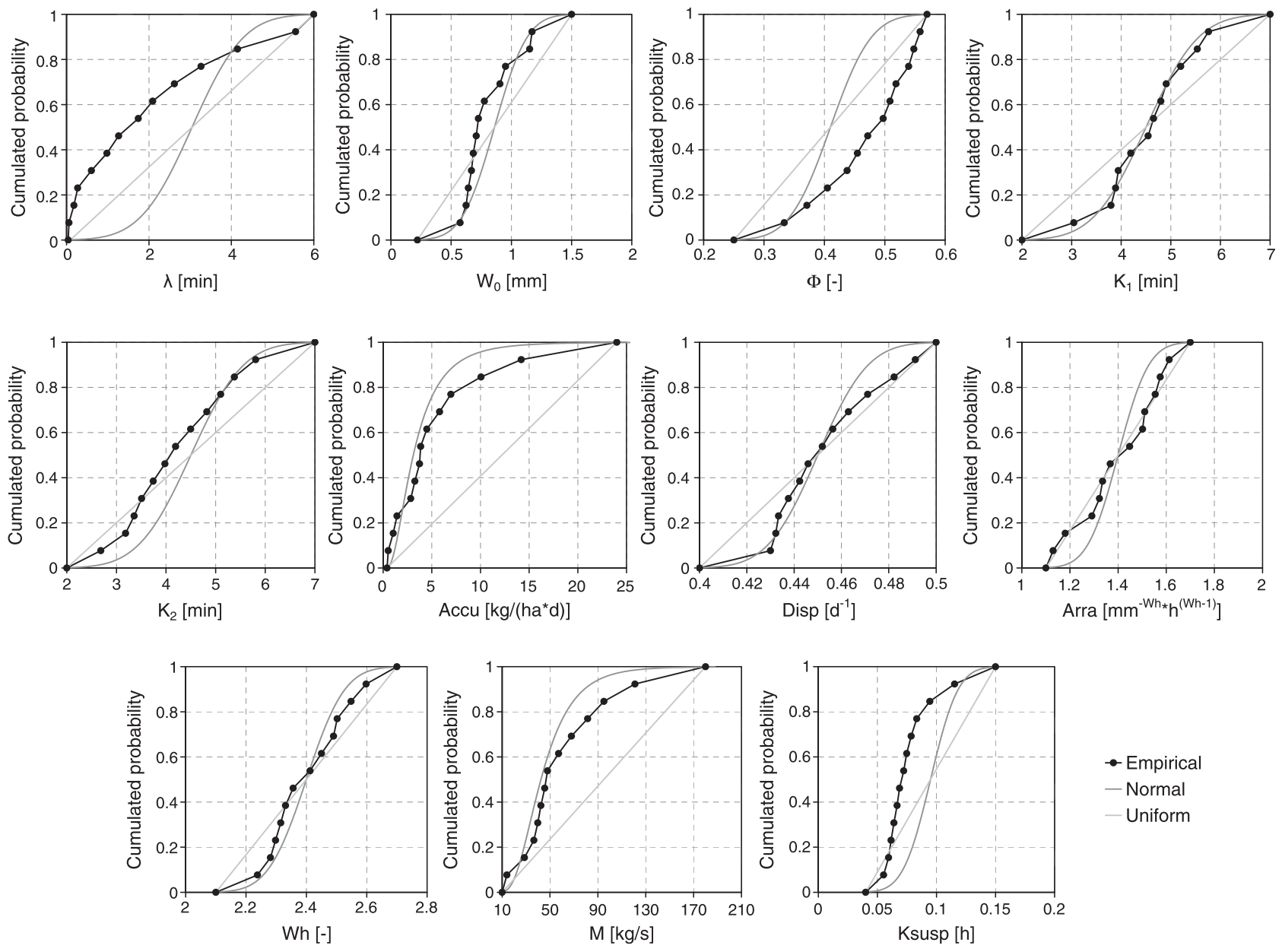

Fig. 1. Empirical frequency distributions of some model parameters.

- After the second Bayesian update, all parameters converge to similar distributions regardless of the prior distributions. This result leads us to conclude that the Bayesian approach is robust; further, if new data are provided, a few additional updates may lead to essentially equal distributions.

- The influence of the prior distribution is mitigated by the successive updates; however, it can be relevant in the first steps of Bayesian analysis depending on the distance between the prior hypothetical distribution and the unknown "real" one. Parameters like ARRA, KSUSP (Fig. 2) and $W_{0}$ (Fig. 3b, d, and f) show that, if a uniform distribution is selected, the final obtained distribution (after two Bayesian updates) is less neat than the other prior hypotheses. Similar results may be obtained when the expected value in the prior distribution is far from the unknown "real" one, as is the case for Arra when a normal distribution is selected (Fig. 3c).

- The use of empirical distributions always provides for smaller updates in terms of the resulting distributions; such a finding emphasises the importance of having significant prior knowledge at the beginning of the Bayesian uncertainty analysis.

Fig. 4 shows the uncertainty bands based on the analysis of hydrographs at the sewer outflow. Fig. 4a-c shows the first Bayesian update stage (using data from six events only) in the three knowledge scenarios, empirical, uniform, and normal/log-normal distributions, respectively. In Table 3 , the results are summarised in terms of the average and the maximum band width and the greatest distance between the maximum efficiency simulation and the 5\% (Dmax_5) and 95\% band limits (Dmax_95) for the sewer system outflow discharges. Analysis of these results enables us to draw the following conclusions:

- For the first step of the Bayesian approach, the effect of empirical prior knowledge is made evident by comparing Fig. 4a with Fig. 4b, the uniform distribution, and Fig. 4c, the normal/log-normal distributions. In particular, the use of a uniform distribution in Fig. 4b creates larger uncertainty bands, especially in the decreasing limb of the hydrograph, even if the widths of the uncertainty band near the respective peaks are similar. Indeed, the maximum band width, Dmax_5, and Dmax_95 are 0.334, 0.206, and 0.294 (Table 3), respectively. The larger uncertainty bands caused by the use of a uniform distribution are due to the fact that uniform distributions contain less information, and therefore more uncertainty, than other distributions with the same minimum and maximum. Such results are consistent with previous studies (Benedetti et al., 2008), which suggest that in case of scarce knowledge about model parameters, it is advisable to adopt uniform distributions to avoid underestimation of the model prediction uncertainties.

- The use of normal distributions results in narrower uncertainty bands with respect to both uniform distributions and empirical ones. As a matter of a fact, for normal distributions, in the first step of the Bayesian analysis, some measured points fall outside the uncertainty bands, drawing attention to the possibility of model rejection. However, in the second step the uncertainty bands encompass all measured points, ensuring the adaptability of the model for the selected case study. 
(a)

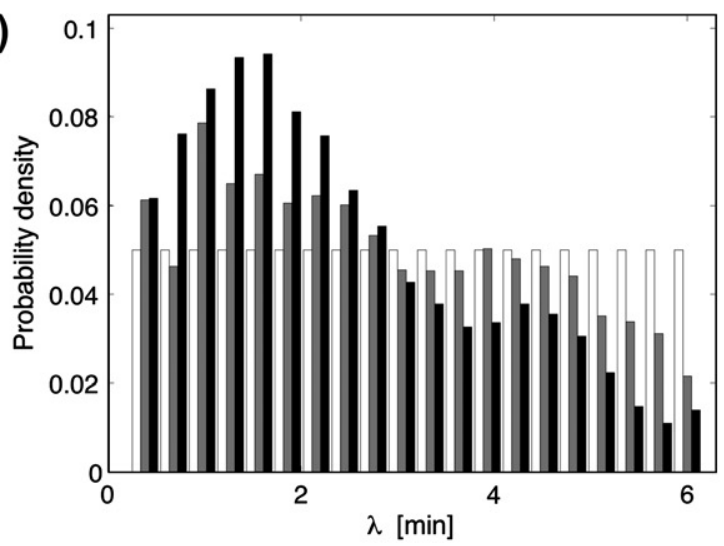

(c)

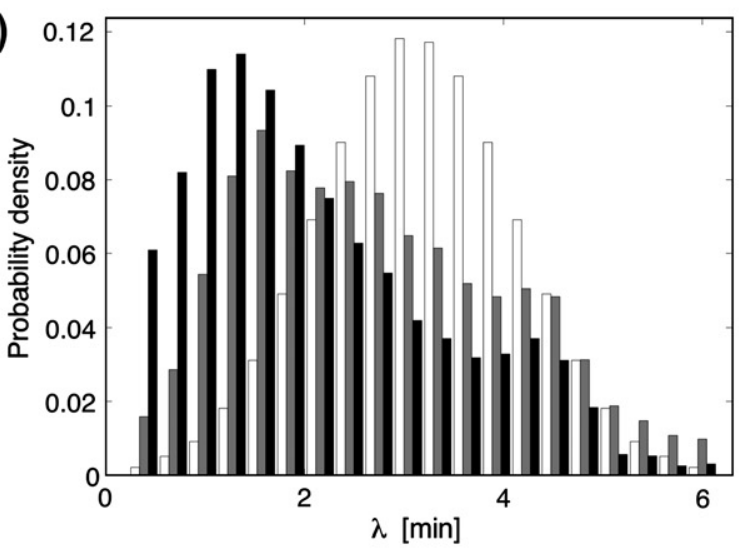

(e)

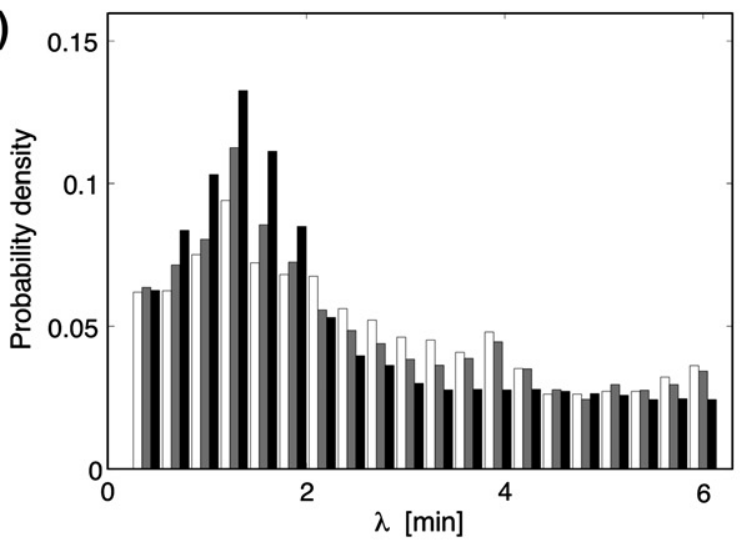

(b)

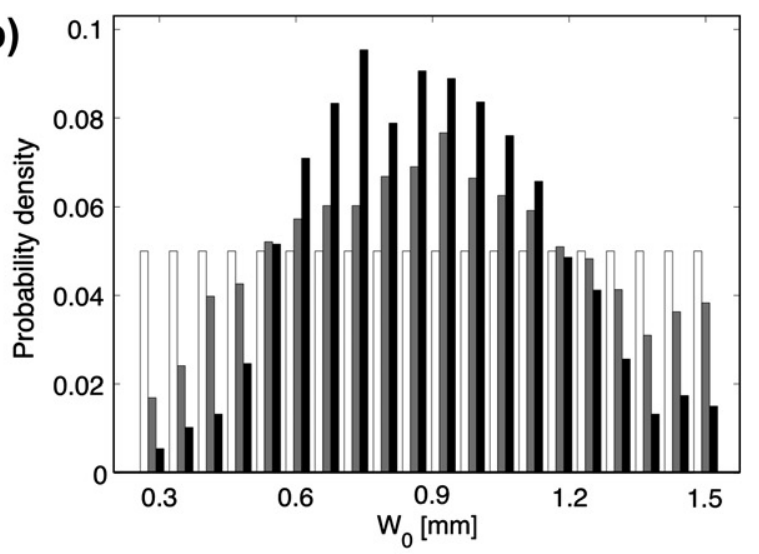

(d)

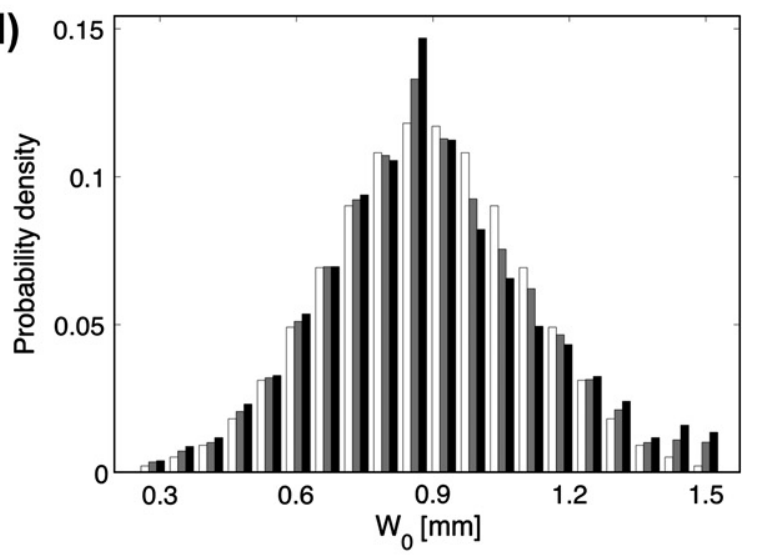

(f)

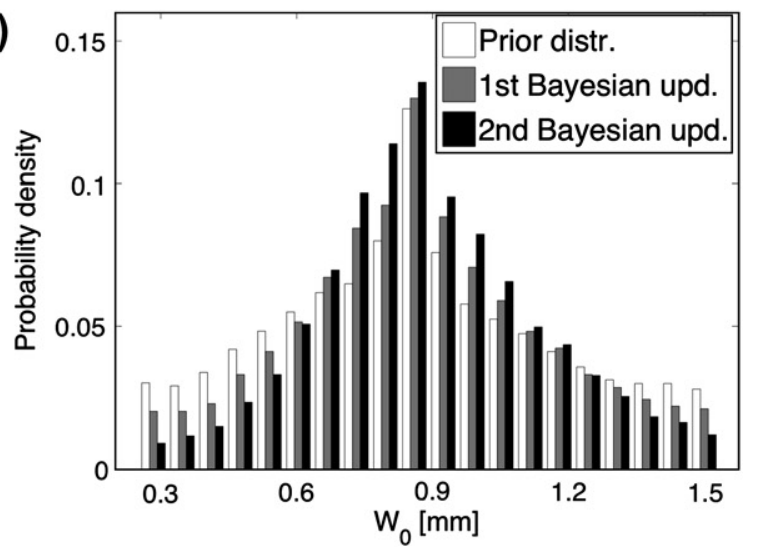

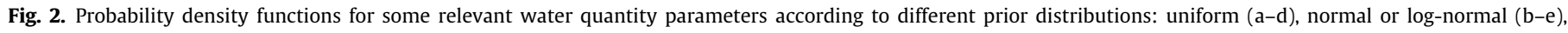
empirical (c-f).

This last aspect highlights the importance of initial assumptions that may lead to a wrongful rejection of a specific set of model parameters and to the possible underestimation of uncertainty in the model results. This result is mainly due to the shape of normal prior distributions, a characteristic that caused a larger densification of parameter values around the median of the variation range with respect to the empirical one. The results from the second Bayesian update phase (Fig. $4 \mathrm{~d}-\mathrm{f}$ ) show smaller differences between the three knowledge scenarios, thus demonstrating that the Bayesian approach is influenced by initial assumptions almost in the earliest phases of the procedure. Whenever progressively new data become available, the subsequent update steps modify the distributions of the model parameters to the point that prior knowledge is only marginally relevant to the uncertainty analysis.
Fig. 5 and Table 4 shows similar results for the water quality aspects. Uncertainty bands are proportionally higher for the quality results than for quantity parameters, confirming the higher uncertainty connected with water quality modelling reported in other previous studies (Freni et al., 2009b; Willems, 2008; Mannina, 2005; Ashley et al., 1999; Harremoës, 1988). Additionally, in this case, the use of general literature information for gaining prior knowledge (third scenario, Fig. $5 \mathrm{c}$ and $\mathrm{f}$ ) is not advisable because it may lead to the wrong estimation of uncertainty bands and the possibility of dropping modelling hypotheses because too many measured points fall outside the uncertainty bands. Uniform distributions always provide wider uncertainty bands; however, the tendency of posterior distributions (Fig. 5e and f) to be similar after the second Bayesian update can reassure the operator about the reliability of the Bayesian approach independently from prior 
(a)

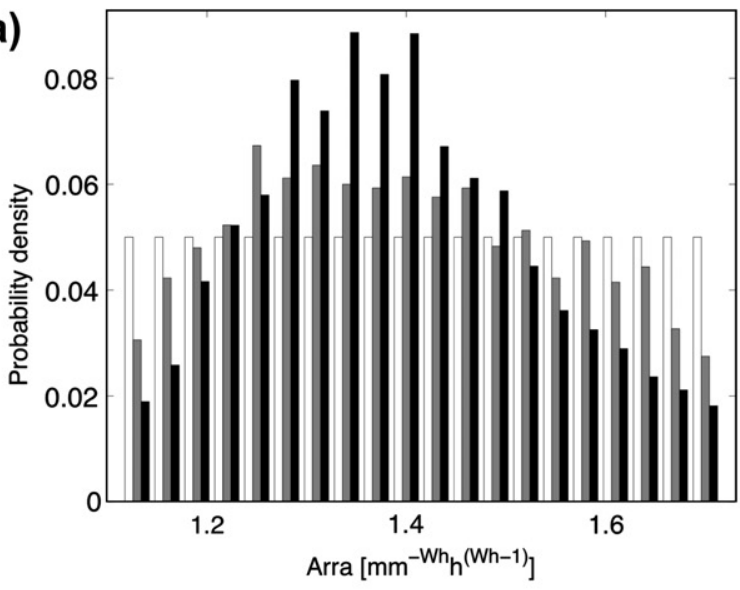

(c)

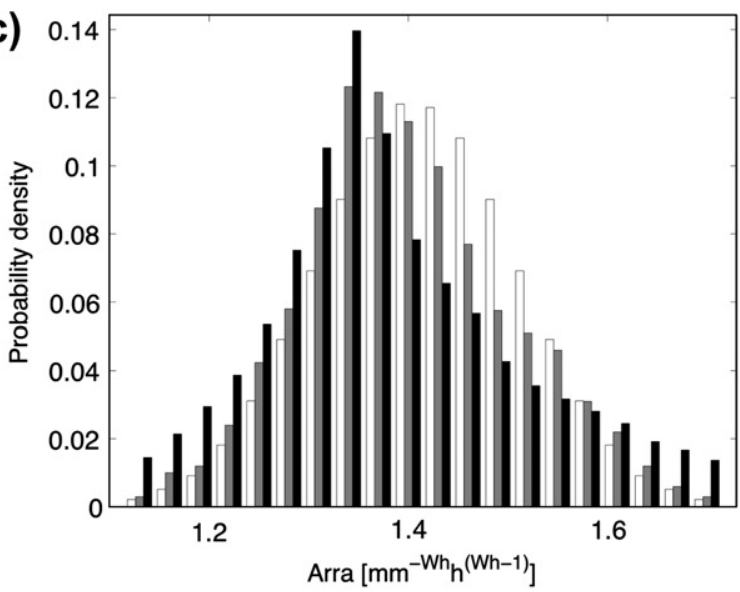

(e)

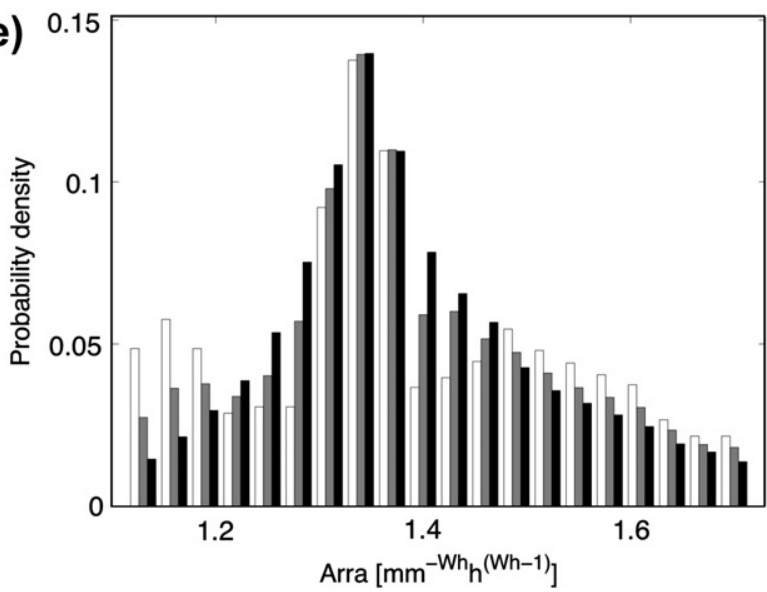

(b)

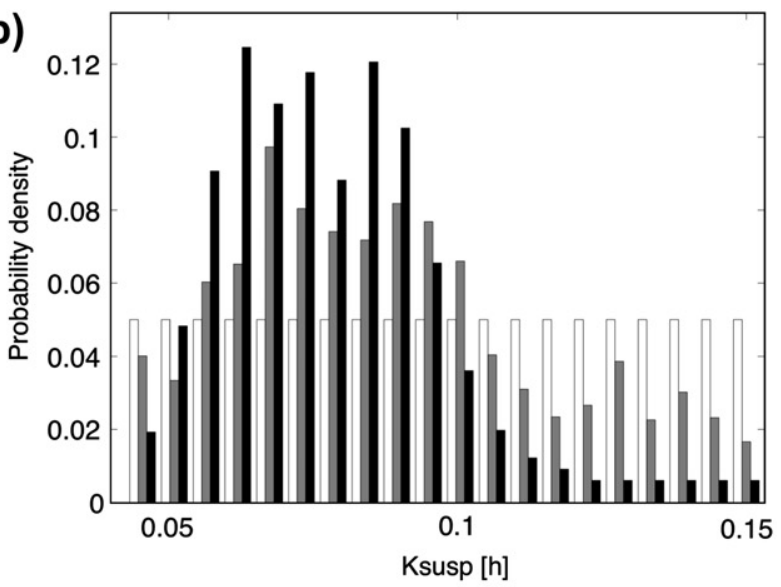

(d)

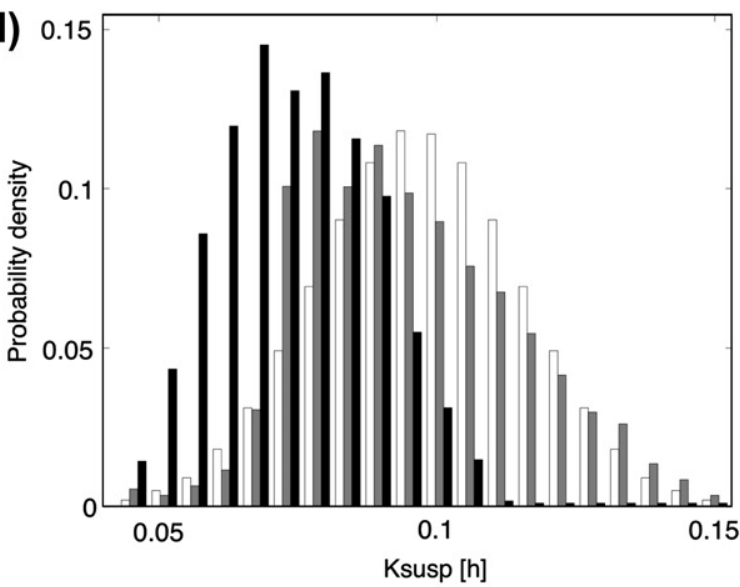

(f)

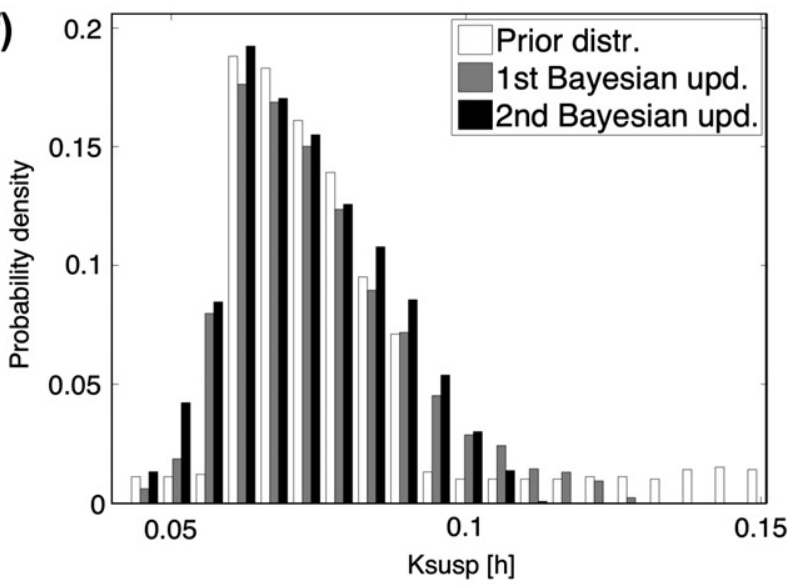

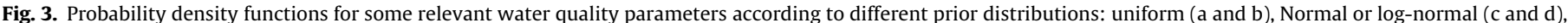
Empirical (e and f).

hypotheses. The drawback of such a hypothesis is the slowness of the learning process, but that can be neglected if the selected case study is monitored systematically and new knowledge is progressively available for Bayesian update. One peculiar aspect of water quality model analysis pertains to the width of the uncertainty bands in the decreasing limb of the pollutograph, a result that seems largely affected by the prior distribution of the parameters.

This behaviour may be influenced by the variability of the erosion coefficient, $M$, which can allow erosion of sediment mass during the whole rainfall event or only at the beginning.

\section{Conclusions}

This paper presented an analysis of the effect of prior knowledge on Bayesian uncertainty analysis. Three knowledge scenarios have been considered, assuming, respectively, no prior knowledge, general knowledge about parameter values and their variation ranges, and a reference scenario in which knowledge has been derived from available monitored events.

This study demonstrated that the impact of prior knowledge is limited to the initial update phases of the Bayesian approach. The following steps are progressively less influenced by such assump- 

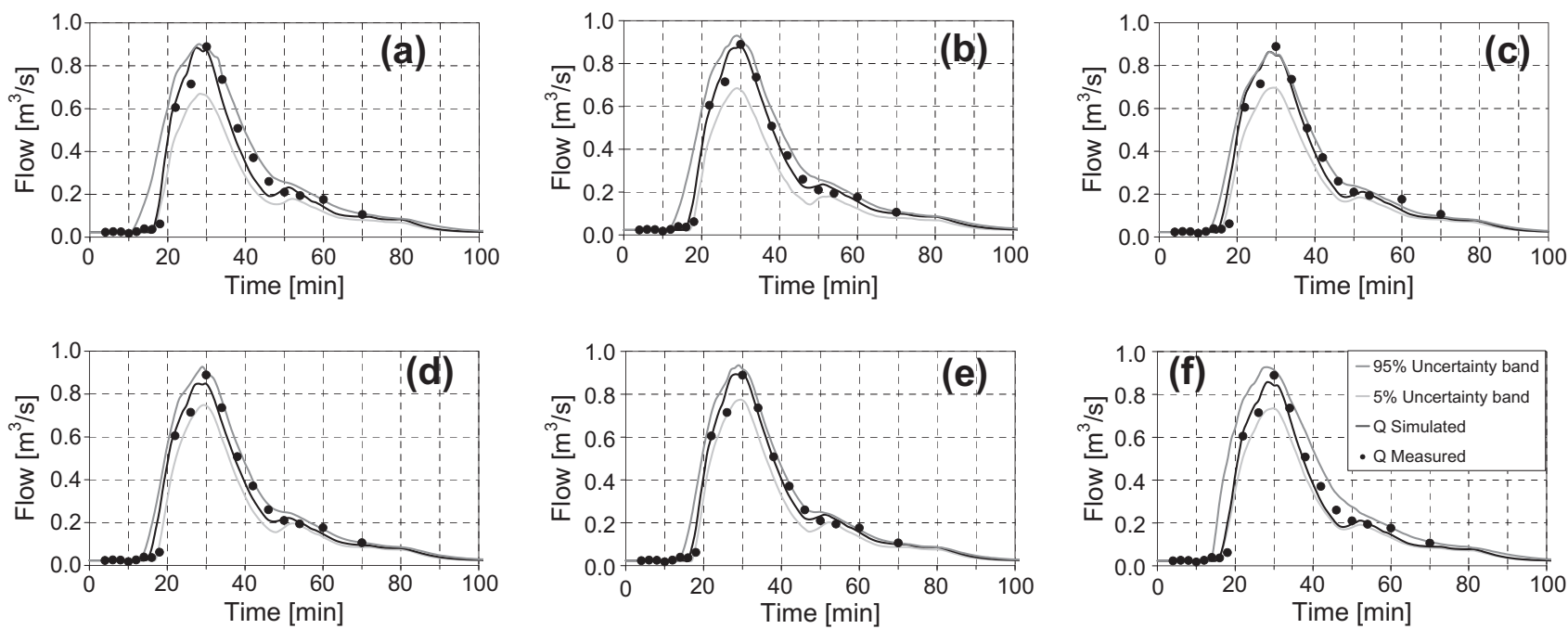

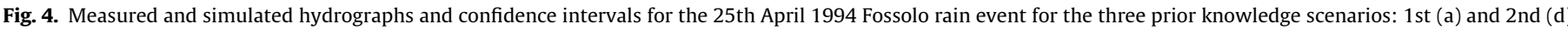
Bayesian update adopting empirical distributions; (b and e) adopting the uniform distributions; (c and f) adopting the normal/log-normal distributions.

Table 3

Uncertainty assessment results for SS outfall discharge $\left(\mathrm{m}^{3} / \mathrm{s}\right)$ in the three knowledge scenarios

\begin{tabular}{|c|c|c|c|c|c|c|c|c|}
\hline \multirow[t]{2}{*}{ Knowledge scenario } & \multicolumn{2}{|c|}{ Av. band width } & \multicolumn{2}{|c|}{ Max band width } & \multicolumn{2}{|l|}{ Dmax_5 } & \multicolumn{2}{|l|}{ Dmax_95 } \\
\hline & 1st stage & 2nd stage & 1st stage & 2nd stage & 1st stage & 2 2nd stage & 1st stage & 2nd stage \\
\hline No prior knowledge - uniform distributions & 0.173 & 0.151 & 0.334 & 0.248 & 0.206 & 0.193 & 0.294 & 0.223 \\
\hline General knowledge - normal/log-normal distributions & 0.121 & 0.142 & 0.216 & 0.239 & 0.173 & 0.205 & 0.135 & 0.188 \\
\hline Knowledge based on calibrated event - empirical distributions & 0.160 & 0.135 & 0.321 & 0.224 & 0.232 & 0.183 & 0.257 & 0.192 \\
\hline
\end{tabular}
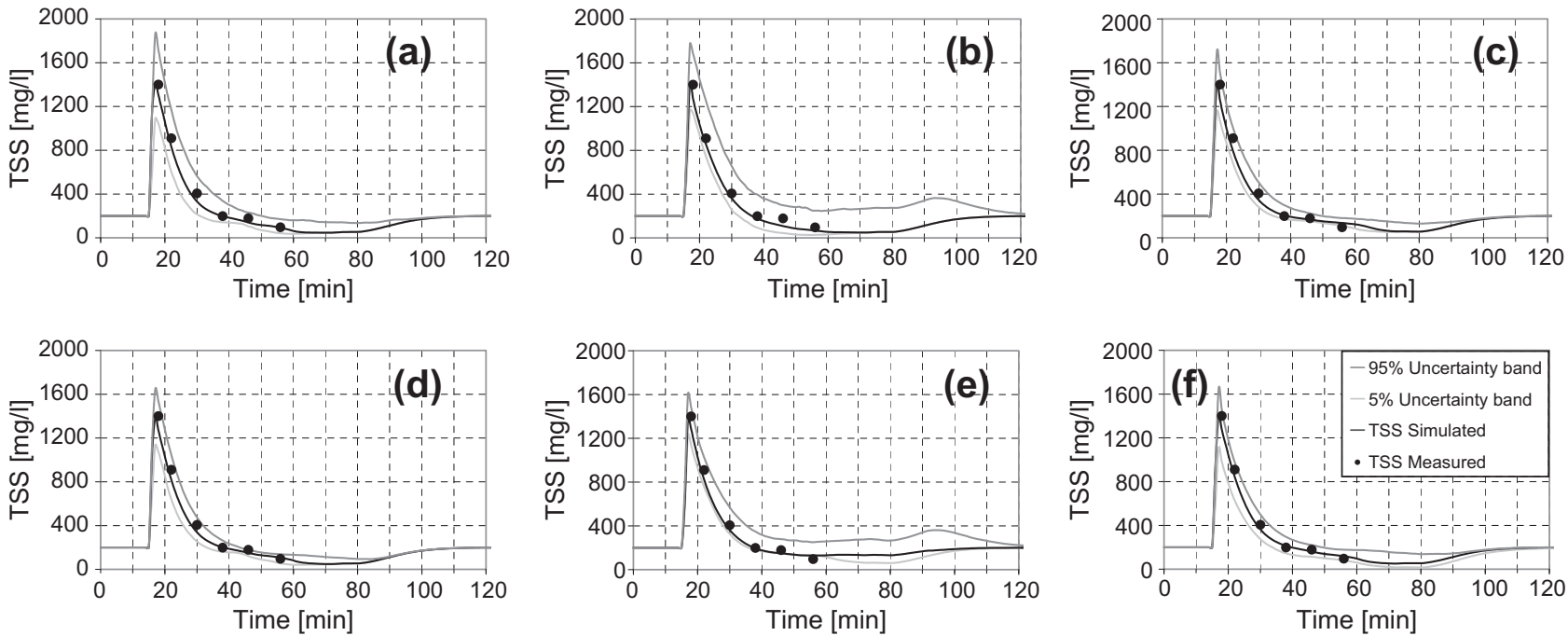

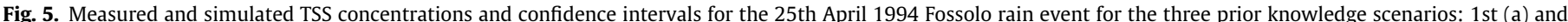
2nd (d) Bayesian update adopting empirical distributions; (b and e) adopting the uniform distributions; (c and f) adopting the normal/log-normal distributions.

Table 4

Uncertainty assessment results for SS outfall TSS concentration $(\mathrm{mg} / \mathrm{l})$ in the three knowledge scenarios

\begin{tabular}{|c|c|c|c|c|c|c|c|c|}
\hline \multirow[t]{2}{*}{ Knowledge scenario } & \multicolumn{2}{|c|}{ Av. band width } & \multicolumn{2}{|c|}{ Max band width } & \multicolumn{2}{|l|}{ Dmax_5 } & \multicolumn{2}{|l|}{ Dmax_95 } \\
\hline & 1st stage & 2nd stage & 1st stage & 2nd stage & 1st stage & 2nd stage & 1st stage & 2nd stage \\
\hline No prior knowledge - uniform distributions & 185.2 & 154.3 & 854.4 & 567.6 & 556.6 & 352.2 & 367.4 & 332.2 \\
\hline General knowledge - normal/log-normal distributions & 104.9 & 131.1 & 544.6 & 543.5 & 248.2 & 345.6 & 296.4 & 252.6 \\
\hline Knowledge based on calibrated event - empirical distributions & 156.6 & 97.5 & 731.1 & 511,8 & 550.1 & 348.3 & 348.2 & 304.3 \\
\hline
\end{tabular}


tions, thus ensuring that uncertainty analysis becomes progressively more objective, if sufficient amounts of data are provided.

The availability of prior knowledge based on previously monitored events presents an ideal condition for analysis because the initial updates of the parameters' probability distributions are much lower than the other cases.

Apart from this aspect, prior knowledge and the selection of appropriate prior parameter distributions can impact the selection and validation of the adopted modelling structure. This procedure is usually based on initial model performance assessment and, as demonstrated in the study, a wrong assumption of the operator about the prior parameter distribution may lead to the rejection of a modelling structure that might have been accepted if prior knowledge about parameters was available. This last consideration leads to the conclusion that the uniform distribution should be preferred unless relevant information about parameters is available in the specific case study. The adoption of other distributions may also cause initial underestimation of uncertainty bands, thus generating excessive confidence in the model results. As demonstrated in the study, this aspect is then corrected in the following update steps, but this behaviour may be interpreted by inexpert users as the poor ability of the model to learn from new available data.

\section{Acknowledgements}

The authors gratefully acknowledge Prof. S. Artina and Dr. M. Maglionico (D.I.S.T.A.R.T. - University of Bologna, IT) for providing data of the Fossolo catchment. The authors would like to thank Prof. A. Montanari for the proficuous discussion and suggestions during the review of the paper.

\section{References}

APHA., 1995. Standard Methods for Examination of Water and Wastewater. APHA AWWA and WPCF, Washington DC, USA.

Artina, S., Maglionico, M., Marinelli, A., Raffaelli, G., Anzalone, C., Lanzarini, S. Guzzinati, E., 1997. Le misure di qualità nel bacino urbano Fossolo. L'acqua 2, 17-25.

Ashley, R.M., Bertrand-Krajewski, J-L., Hvitved-Jacobsen, T., Verbanck, M. (2004). Solids in Sewers:Characteristics, Effects and Control of Sewer Solids and Associated Pollutants. Scientific and Technical Report No. 14, IWA Publishing, London, UK.

Ashley, R.M., Hvitved-Jacobsen, T., Bertrand-Krajewski, J.-L., 1999. Quo vadis sewer process modelling? Water Science and Technology 39 (9), 9-22.

Bates, B.C., Campbell, E.P., 2001. A Markov chain Monte Carlo scheme for parameter estimation and inference in conceptual rainfall-runoff modeling. Water Resources Research 37 (4), 937-947.

Bayes, T., 1763. An essay towards solving a problem in the doctrine of chances Philosophical Transactions of the Royal Society of London 53, 370-418.

Beck, M.B., 1987. Water quality modeling: a review of the analysis of uncertainty. Water Resources Research 23 (8), 1393-1442.

Benedetti, L., Bixio, D., Claeys, F., Vanrolleghem, P.A., 2008. Tools to support a model-based methodology for emission/immission and benefit/cost/risk analysis of wastewater systems that considers uncertainty. Environmental Modelling \& Software 23, 1082-1091.

Bertrand-Krajewski, J.L., Barraud, S., Bardin, J.P., 2002. Uncertainties, performance indicators and decision aid applied to storm water facilities. Urban Water 4 163-179.

Beven, K.J., Binley, A.M., 1992. The future of distributed models - model calibration and uncertainty prediction. Hydrological Processes 6 (3), 279-298.

Box, G.E.P., Cox, D.R., 1982. An analysis of transformations revisited, rebutted Journal of the American Statistical Association 77 (377), 209-210.

Chin, D.A., 2009. Predictive uncertainty in water-quality modeling. Journal of Environmental Engineering 135 (12), 1315-1325.

Deletic, A., Dotto, C., Fletcher, T.D., McCarthy, D.T., Bertrand-Krajewski, JL., Rauch, W., Kleidorfer, M., Freni, G., Mannina, G., Tait, S., 2009. Defining uncertainties in modelling of urban drainage systems. In: Proc., 8th International Conference on Urban Drainage Modelling. Tokyo, 7-11 September 2009. Ed. Global Center of Excellence for Sustainable Urban Regeneration, Tokyo.

Freni, G., Mannina, G., Viviani, G., 2008. Uncertainty in urban stormwater quality modelling: the effect of acceptability threshold in the GLUE methodology. Water Research 42 (8-9), 2061-2072.

Freni, G., Mannina, G., Viviani, G., 2009a. Urban runoff modelling uncertainty: comparison among Bayesian and pseudo-Bayesian methods. Environmental Modelling Software 24, 54-62.
Freni, Mannina, G., Viviani, G., 2009b. Assessment of data availability influence on integrated urban drainage modelling uncertainty. Environmental Modelling Software 24 (10), 1171-1181.

Gallagher, M.R., Doherty, J., 2007. Parameter interdependence and uncertainty induced by lumping in a hydrologic model. Water Resources Research 43 (5), W05421.

Harremoës, P., 1988. Stochastic models for estimation of extreme pollution from urban runoff. Water Resources 22 (8), 1017-1026.

Harremoës, P., Madsen, H., 1999. Fiction and reality in the modelling world-balance between simplicity and complexity, calibration and identifiability, verification and falsification. Water Science and Technology 39 (9), 1-8.

Iman, R.L., Helton, J.C., Campbell, J.E., 1981. An approach to sensitivity analysis of computer models, Part 1 . Introduction, input variable selection and preliminary variable assessment. Journal of Quality Technology 13 (3), 174-183.

Jaynes, E.T., 1968. Prior PROBABILITIES. IEEE Transactions on Systems Science and Cybernetics 4 (3), 227-241.

Kanso, A., Chebbo, G., Tassin, B., 2004. Application of MCMC-GSA model calibration method to urban runoff quality modelling. Journal of Reliability Engineering \& System Safety 32 (3), 56-68.

Kanso, A., Chebbo, G., Tassin, B., 2005. Bayesian analysis for erosion modelling of sediments in combined sewer systems. Water Science and Technology 52 (5), 135-142.

Kavetski, D., Kuczera, G., Franks, S.W., 2006a. Bayesian analysis of input uncertainty in hydrological modeling: 1. Theory. Water Resources Research 42 (3), W03407. Kavetski, D., Kuczera, G., Franks, S.W., 2006b. Bayesian analysis of input uncertainty in hydrological modeling: 2. Application. Water Resources Research 42 (3), W03408.

Kottegoda, N.T., Rosso, R., 2008. Applied Statistics for Civil and Environmental Engineers. Wiley \& Sons Ltd., New York.

Krysanova, V., Hattermann, F., Wechsung, F., 2007. Implications of complexity and uncertainty for integrated modelling and impact assessment in river basins. Environmental Modelling Software 22 (5), 701-709.

Kuczera, G., Parent, E., 1998. Monte Carlo assessment of parameter uncertainty in conceptual catchment models: the Metropolis algorithm. Journal of Hydrology $211,69-85$.

Lindenschmidt, K.-E., Fleischbein, K., Baborowski, M., 2007. Structural uncertainty in a river water quality modelling system. Ecological Modelling 204 (3-4), 289-300.

Mannina, G., 2005. Integrated Urban Drainage Modelling with Uncertainty for Stormwater Pollution Management. PhD thesis, Università di Catania, Italy.

Mannina, Freni, G., Viviani, G., Sægrov, S., Hafskjold, L.S., 2006. Integrated urban water modelling with uncertainty analysis. Water Science and Technology 54 (6-7), 379-386.

Mannina, G., Viviani, G., 2010. An urban drainage stormwater quality model: model development and uncertainty quantification. Journal of Hydrology 381 (3-4), 248-265.

Mantovan, P., Todini, E., 2006. Hydrological forecasting: incoherence of the GLUE methodology. Journal of Hydrology 330, 368-381.

Parchure, T.M., Mehta, A.J., 1985. Erosion of soft cohesive sediment deposits. Journal of Hydrogen Energy 111 (10), 1308-1326.

Refsgaard, Nilsson, B., Brown, J., Klauer, B., Moore, R., Bech, T., Vurro, M., Blind, M., Castilla, G., Tsanis, I., Biza, P., 2005. Harmonised techniques and representative river basin data for assessment and use of uncertainty information in integrated water management. Environment Science Policy 8 (3), 267-277.

Reichert, P., 1997. On the necessity of using imprecise probabilities for modelling environmental systems. Water Science and Technology 36 (5), 149-156.

Reichert, P., Omlin, M., 1997. On the usefulness of overparameterized ecological models. Ecological Modelling 95 (2-3), 289-299.

Ristenpart, E., 1995. Sediment properties and their changes in sewer. Water Science and Technology 25 (8), 1-12.

Schaarup-Jensen, K., Johansen, C., Thorndahl, S., 2005. Uncertainties Related to Extreme Events Statistics of Sewer System Surcharge and Overflow. 10th ICUD, Copenhagen, Denmark, 21-26 August 2005.

Skipworth, P.J., Tait, S.J., Saul, A.J., 1999. Erosion of beds in sewers: model development. Journal of Environment Engineering 125 (6), 566-573.

Thorndahl, S., Willems, P., 2008. Probabilistic modelling of overflow, surcharge and flooding in urban drainage using the first-order reliability method and parameterization of local rain series. Water Research 42 (1-2), 455-466.

Thorndahl, S., Beven, K.J., Jensen, J.B., Schaarup-Jensen, K., 2008. Event based uncertainty assessment in urban drainage modelling, applying the GLUE methodology. Journal of Hydrology 357 (3-4), 421-437.

Thyer, M., Kuczera, G., Wang, Q.J., 2002. Quantifying parameter uncertainty in stochastic models using the Box-Cox transformation. Journal of Hydrology 265 (1-4), 246-257.

Willems, P., 2000. Probabilistic Immission Modelling for Receiving Surface Waters. Ph.D. Thesis. Faculty of Engineering, Katholieke Universiteit Leuven, Leuven, Belgium.

Willems, P., 2008. Quantification and relative comparison of different types of uncertainties in sewer water quality modeling. Water Research 42 (13), 35393551.

Willems, P., 2009. Parsimonious model for combined sewer overflow pollution. Journal of Environmental Engineering 136 (3), 316-325.

Willems, P., Berlamont, J., 2002. Probabilistic emission and immission modelling: case-study of the combined sewer-WWTP-receiving water system at Dessel (Belgium). Water Science and Technology 45 (3), 117-124.

Yang, J., Reichert, P., Abbaspour, K.C., Xia, J., Yang, H., 2008. Comparing uncertainty analysis techniques for a SWAT application to the Chaohe Basin in China. Journal of Hydrology 358 (1-2), 1-23. 